\title{
Modelling and thermography measurements of thermal properties of nonwoven
}

\author{
by M. Michalak ${ }^{1}$, B. Więcek², I. Krucińska ${ }^{1}$ \\ (1) Department of Textile Metrology, Technical University of Łódź. Poland \\ (2) Computer Thermography Group, Institute of Electronics, Technical University of Łódź, \\ Poland
}

\section{Abstract}

By means of a thermography method we studied the thermal parameters of nonwovens manufactured from hemp fibres, chemical fibres and with an addition of electrically conducting fibres. In textile, such investigations using the thermo wave method was applied for the first time.

\section{Introduction}

Barrier properties of various textile materials, understood as the properties of suppressing the energy of electromagnetic wave, have been thoroughly studied in recent years. The interest in the subject results from the fact that in recent years man has been more often exposed to harmful effects of electromagnetic radiation. The problem became more acute when mobile phones appeared in our everyday life. To protect human organism from radiation, different types of screening are often used. Producers of screening materials show interest in textiles due to their usable, ecological, health, and economic qualities. More and more frequently bast fibres are studied, e.g. hemp fibres. Paper [1] shows that hemp fibres can screen electromagnetic radiation of high frequency (up to $300 \mathrm{GHz}$ ).

Nonwovens are technologically easiest, and thus economically justified, to use for screening purposes (not only for rooms but even for mobile phones casing). Nonwovens are materials produced from a mass of fibres formed into a web or filaments made into a flat product by means of various binding agents (sized non-wovens) or mechanically - by needling or sewing (needled or sewn non-wovens). A previous paper [2] made an attempt at studying barrier properties against electromagnetic fields of the range of infrared radiation of needled non-wovens produced from chemical fibres, hemp fibres of alternative physical properties and non-wovens produced from hemp fibres with an addition of electrically conducting fibres. In the studies a thermography method was used. The surface of the studied non-woven sample was exposed to infrared radiation during $100 \mathrm{~s}$. Distribution of temperature on the other surface of the sample was registered during the exposition time and after it for the time of $100 \mathrm{~s}$. Results of this experiment were compared with the results of temperature distribution measurements obtained at registration of temperature distribution on the exposed surface. Analysis of the obtained results let us draw a conclusion that hemp fibres have better barrier properties than the studied chemical fibres.

This paper presents further studies on the above mentioned materials. The thermal parameters are determined. The paper presents the first results of studies using the thermal wave method [3]. So far this method has not been used for textile products.

\section{Thermal model and simulation results}

In order to simulate the transient thermal process in the textile material, we have taken the sample presented in Fig. 1. Power was delivered as a step function of heat flux $\phi_{0}=1 \mathrm{~kW} / \mathrm{m}^{2}$ on the left of the nonwoven. The one dimensional case is considered, and the 
heat flows from left to right along the $x$ axis. Free convection from both sides of the sample is assumed with the heat transfer coefficient $h=5 W\left(m^{2} \cdot K\right)$.

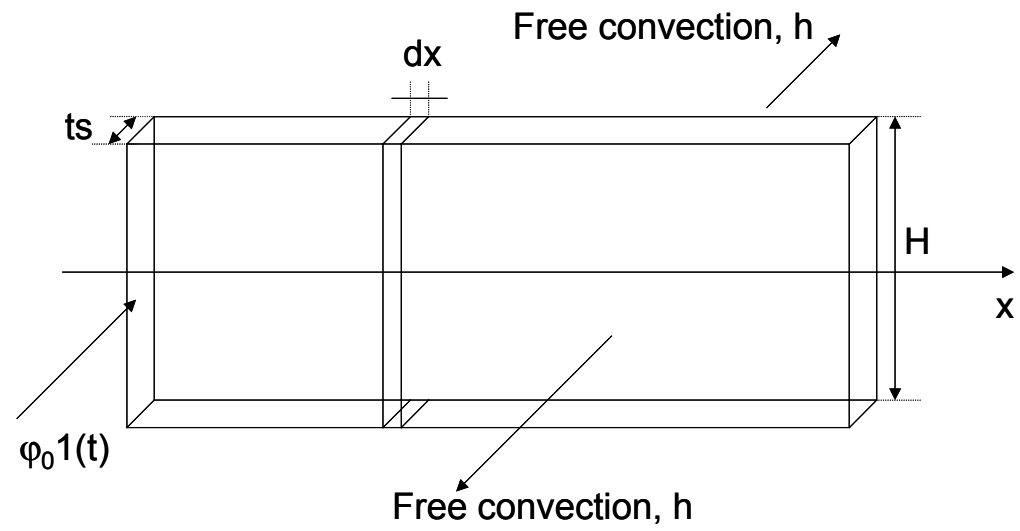

Fig. 1. Nonwoven sample taken for modelling

The simplified thermal model is expressed as:

$$
\frac{\partial^{2} T}{\partial x^{2}}-\frac{T}{L^{2}}=\frac{1}{a} \frac{\partial T}{\partial t}
$$

where: diffusivity $a=\frac{\lambda}{c_{p} \rho}$, and characteristic length $L=\sqrt{\frac{\lambda t_{s}}{2 h}}$.

Using the Laplace transform we can omit the derivation in time, and it results as:

$$
\begin{aligned}
& \frac{\partial^{2} T}{\partial \mathrm{x}^{2}}-\frac{T}{L^{2}}=s \frac{1}{\mathrm{a}} T \\
& \frac{\partial^{2} T}{\partial \mathrm{x}^{2}}-\frac{T}{L^{2}}=0
\end{aligned}
$$

where: $L^{\prime}(s)=\frac{1}{\sqrt{\frac{1}{L^{2}}+\frac{s}{a}}}$.

Impulse response $T_{\delta}$ is the solution of equation (2) and it takes a form:

$$
T_{\delta}(s)=\frac{\varphi_{0} L^{\prime}}{\lambda} e^{-\frac{x}{L}}
$$

Using inverse Laplace transform (from tables) we can easily find the impulse response as:

$$
T_{\delta}(x, t)=\frac{\varphi_{0}}{\lambda} \sqrt{\frac{a}{\pi t}} e^{-\frac{t a}{L^{2}}-\frac{x^{2}}{4 a t}}
$$

To obtain the step function response we perform the convolution of $T_{\delta}$ and $1(t)$.

$$
T(x, t)=T_{\delta}(x, t) * 1(t)=\int_{0}^{t} T_{\delta}(x, \tau) 1(t-\tau) d \tau
$$


Simulations were performed for 4 different textile materials with different thermal properties presented in Table 1.

Table 1. Properties of the samples used in the simulation and measurements

\begin{tabular}{|c|c|c|c|c|}
\cline { 2 - 5 } \multicolumn{1}{c|}{} & Sample 1 & Sample 2 & $\begin{array}{c}\text { Sample 3 } \\
\text { With electrically } \\
\text { conducting fibres }\end{array}$ & Sample 4 \\
\hline $\begin{array}{c}\text { Thermal } \\
\text { conductivity } \\
\lambda[\mathrm{W} /(\mathrm{m} \cdot \mathrm{K})\end{array}$ & 0.0487 & 0.0578 & 0.0618 & 0.0352 \\
\hline $\begin{array}{c}\text { Thermal diffusivity } \\
\mathrm{A}\left[\mathrm{m}^{2} / \mathrm{s}\right]\end{array}$ & $0.415 \mathrm{e}-6$ & $0.275 \mathrm{e}-6$ & $0.459 \mathrm{e}-6$ & $0.624 \mathrm{e}-6$ \\
\hline $\begin{array}{c}\mathrm{Effusivity} \\
\mathrm{e}\left[\mathrm{Ws} \mathrm{s}^{1 / 2} /\left(\mathrm{m}^{2} \mathrm{~K}\right)\right.\end{array}$ & 75.6 & 110 & 91.2 & 44.2 \\
\hline $\begin{array}{c}\text { Thickness } \\
{[\mathrm{m}]}\end{array}$ & 0.00768 & 0.00596 & 0.00737 & 0.00378 \\
\hline
\end{tabular}

The results are presented below. At first, we display the temperature distribution in the sample as a function of $x$. (Fig. 2). As expected the temperature decreases along the $x-$ axis, and it rises with time. The different thermal properties of the material used for simulation causes the differences of temperature. The aim of this simulation was to confirm how far the temperature difference can be used to evaluate thermal properties of the material, such as conductivity and diffusivity, using the thermal wave method.

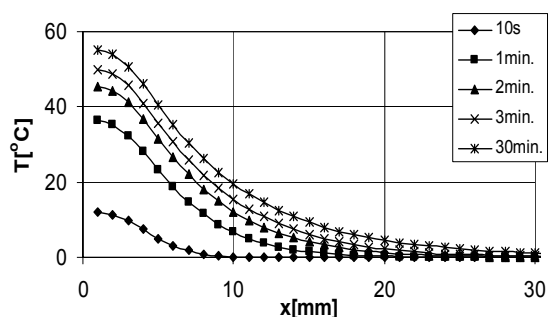

(a)

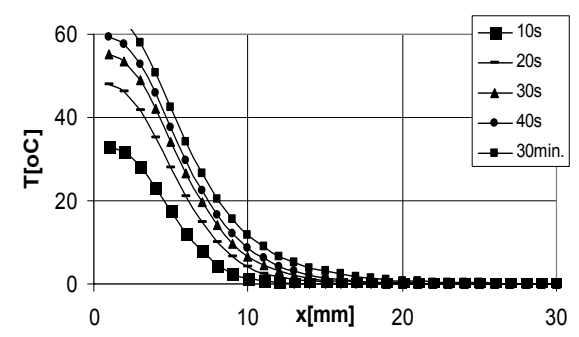

(b)

Fig. 2. Thermal responses for sample 3 (a) and sample 4 (b), curves $T(x)$ for different moments in time

From Fig. 2 one can notice the different thermal responses for different materials. This evident fact is additionally confirmed in Fig. 3, which shows how temperature obtained at the certain moment during transient thermal process correlates with the thermal property of the material. For thermal conductivity we have chosen the temperature close to the beginning of thermal excitation (10s after), while for diffusivity the temperature later (1 $\mathrm{min}$. after) can better differentiate the unknown parameter. It is due to the fact that diffusivity depends on thermal capacity, and after a certain time the temperature differences for different samples are larger. 


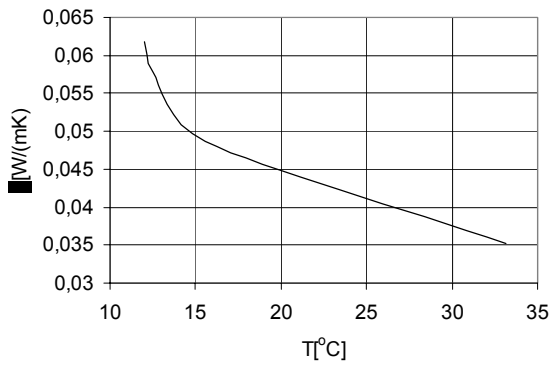

(a)

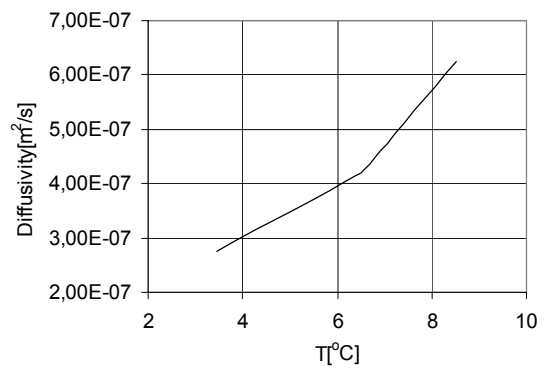

(b)

Fig. 3. Thermal conductivity and diffusivity as a function of temperature obtained for different temperature (a) $t=10$ s and (b) -1 min. after start of the heating process

\section{Experiment}

The purpose of our work was to study the reaction of nonwovens made from hemp fibres to infrared radiation and to compare it with the reaction of similar nonwovens made with addition of electrically conducting fibres and also nonwovens manufactured from blends of chemical fibres. The thermal parameters are determined using pulse thermography.

Three types of needle punched nonwovens were studied. They had similar thickness parameters and the same density of needle punching (80). Nonwovens were manufactured from hemp fibres and alternative chemical fibres of similar morphological characteristics.

- Nonwoven from hemp fibres $60 \mathrm{~mm}$ long. Surface mass of the nonwoven was 750 $\mathrm{g} / \mathrm{m}^{2}$, and its thickness $5.75 \mathrm{~mm}$

- Nonwoven from a blend of polypropylene fibres (PP) $60 \mathrm{~mm}$ long and linear density 6 dtex and polyester fibres (PET) $57 \mathrm{~mm}$ long and linear density 6.7 dtex. Percentage of the blend was $40 / 60$. Surface mass was $625 \mathrm{~g} / \mathrm{m}^{2}$ and thickness $7.1 \mathrm{~mm}$.

- Nonwoven manufactured in the form of „a sandwich”, in which a layer of electrically conducting fibres made on the base of polyacrylonitrile fibres is placed between two layers of hemp fibres. The thickness of the nonwoven was $7.2 \mathrm{~mm}$.

Samples of the size $100 \mathrm{~mm} \times 100 \mathrm{~mm}$ were made from these nonwovens.

The measurements were performed on a measurement stand where a source of infrared radiation ( $3 \mathrm{~kW}$ lamp) was located on one side of a sample while a thermography camera monitored the other side of it. Thus, the temperature field on the non-irradiated sample could be measured. The sample was perpendicular to the optical axis of the thermography camera. The distance between the source of radiation and the sample and from the sample to the camera was the same $(40 \mathrm{~cm})$.

A block diagram of the measuring position is shown in figure 4. 

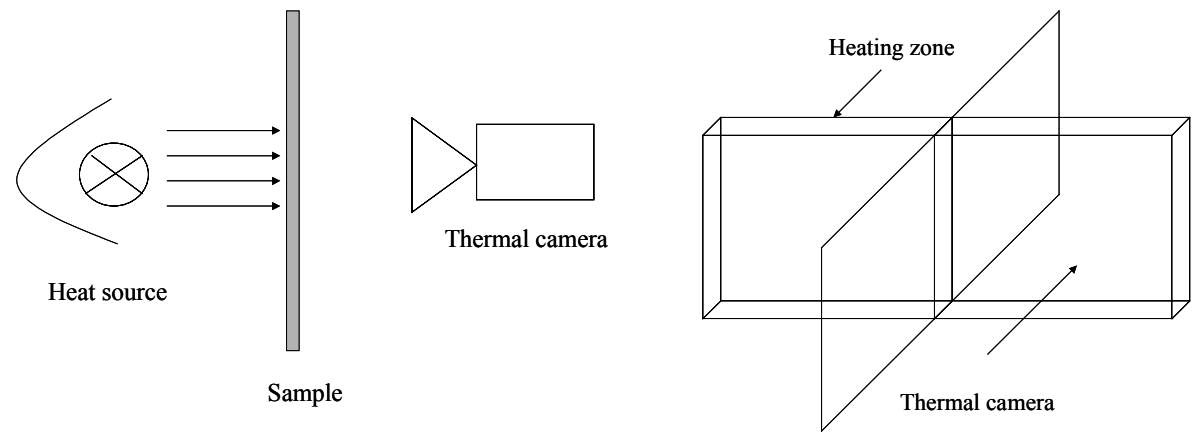

Fig. 4. Experiment setup

The surface of the sample was divided into two areas. One was irradiated by step function heat flux, while the other one was shielded to reduce the influence of reflections from the lamps. The temperature distribution was observed in order to derive thermal properties of the textile materials. The measurements were performed during the cooling down process. The heating and cooling curves should have the same time constant due to the linearity of the investigated system, and in that sense the simulations correspond to the measurements.

An example of a cooling process is presented in Figs. $5-6$. It is noticeable that the temperature decay depends on thermal time constant, which is different for different samples.

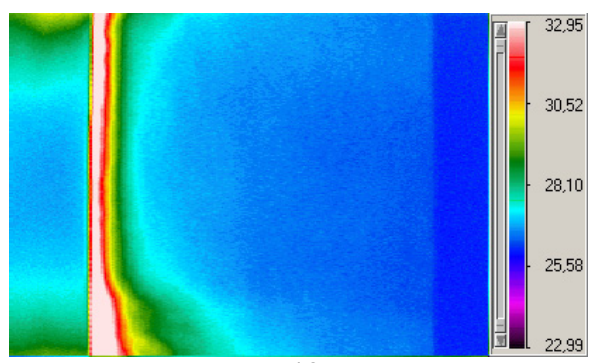

$\mathrm{t}=10 \mathrm{~s}$

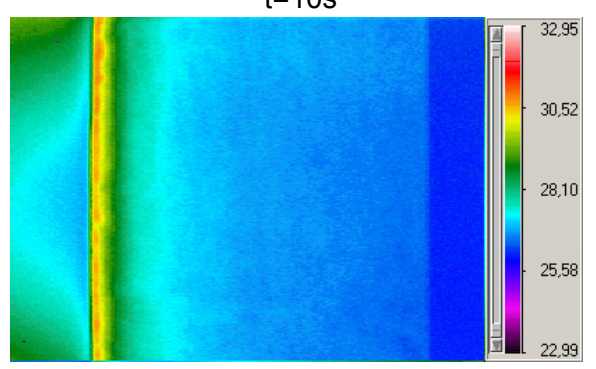

$\mathrm{t}=2 \min$.

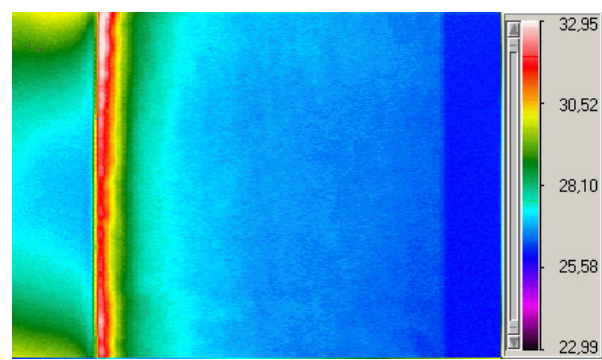

$\mathrm{t}=1 \mathrm{~min}$.

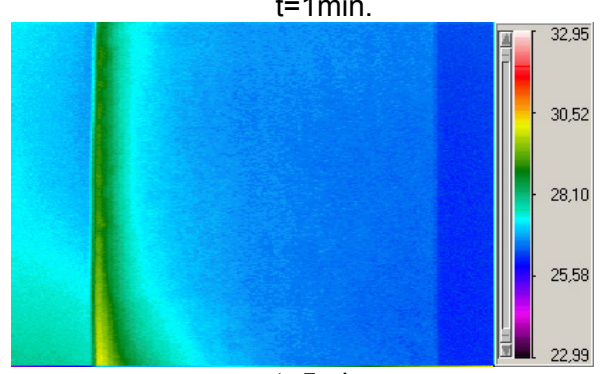

$\mathrm{t}=5 \mathrm{~min}$.

Fig. 5. Temperature distribution in time for sample 3 (nonwovens with electrically conducting fibres) 


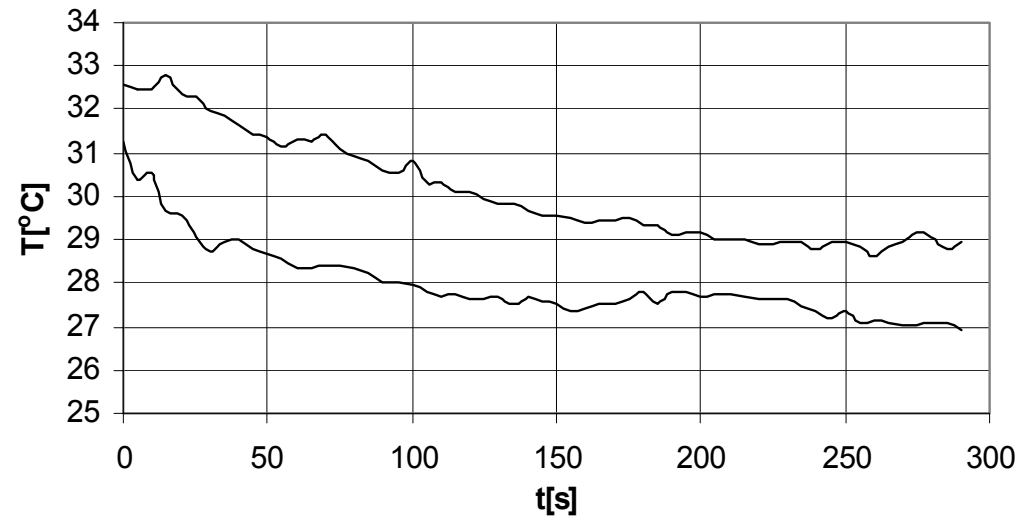

Fig. 6. Experiment results, sample 3 - upper curve, sample 4 - bottom curve

\section{Conclusions}

Results obtained in this study confirm the thesis that pulse thermography is useful to evaluate thermal properties of textile materials. From the decay of temperature during the cooling down process we can differentiate materials with different thermal conductivities and diffusivities. Thermography investigations should be performed very carefully to reduce parasitic elements having a significant influence on the results, mainly infrared reflection, non-stable ambient conditions, undefined convective heat transfer coefficient, etc.

\section{REFERENCES}

[1] R. Brazis, J. Czekalski, D. Kozakiewicz, M. Michalak, M. Stasiak. The Attenuation of Electromagnetic Wave of the Blends Bast/PAN. Fibre and Textile in Eastern Europe. No 2, 2000.

[2] I. Krucińska, M. Michalak. Barrier Properties of Textiles with Hemp Fiber Components. "2nd International Conference „Metrology in Textile Engineering” and NETECOFLAX Workshop, November 2000, Łódź.

[3] M. Michalak, I. Krucińska, B. Więcek, Application of thermography for slow and fast varying thermal processes in textile research, Proc. Quantitative Infrared Termography, QIRT 2000, Reims, July, 2000. 